\section{Paroxysmal alternating skew deviation and nystagmus after partial destruction of the uvula}

\author{
A Radtke, A M Bronstein, M A Gresty, M Faldon, W Taylor, J M Stevens, P Rudge
}

\begin{abstract}
A patient with suspected brain stem glioma involving the area of the left vestibular nuclei and cerebellar peduncle, developed paroxysmal alternating skew deviation and direction changing nystagmus after biopsy of the inferior cerebellar vermis resulting in destruction of the uvula. Between attacks she had right over left skew deviation with asymptomatic right beating horizontal nystagmus. Slow phases of the resting nystagmus showed increasing velocity, similar to congenital nystagmus. At intervals of $40-50$ seconds, paroxysmal reversal of her skew deviation occurred, accompanied by violent left beating horizontal torsional nystagmus lasting 10-12 seconds and causing severe oscillopsia. It is proposed that this complex paroxysmal eye movement disorder results from (1) a lesion in the left vestibular nuclei causing right over left skew and right beating resting nystagmus and (2) a disruption of cerebellar inhibition of vestibular nuclei, causing alternating activity in the vestibular system with intermittent reversal of the skew deviation and paroxysmal nystagmus towards the side of the lesion.
\end{abstract}

(F Neurol Neurosurg Psychiatry 2001;70:790-793)

MRC Human

Movement and

Balance Unit, The

National Hospital for

Neurology and

Neurosurgery, Queen

Square, London

WC1N 3BG, UK

A Radtke

A $M$ Bronstein

M A Gresty

M Faldon

P Rudge

National Hospital for

Neurology and

Neurosurgery

W Taylor

J M Stevens

Correspondence to:

Dr P Rudge

P.Rudge@ion.ucl.ac.uk

Received 22 June 2000 and in revised form

24 January 200

Accepted 14 February 2001

Keywords: skew deviation; periodic alternating nystagmus; uvula

Paroxysmal alternating skew deviation and nystagmus of central origin is rare and may be accompanied by periodic alternating nystagmus. ${ }^{1}$ Various pathologies have been described in these patients including brain stem abscess, ${ }^{2}$ multiple sclerosis, ${ }^{3}$ degenerative vascular disease, ${ }^{4}$ arteriovenous malformation, ${ }^{5}$ and midbrain infarction. ${ }^{6}$ We report on a patient with suspected brain stem glioma who developed paroxysmal alternating skew deviation and direction changing nystagmus after a well defined surgical lesion was made in the uvula, thus indicating that this structure is probably crucial to the development of alternating nystagmus.

\section{Case history}

A 29 year old woman presented with left sided hearing loss in 1992. Six years later she began having episodes of rotational vertigo, together with a feeling of general unsteadiness. Her first vertiginous attack occurred as she was getting out of bed and lasted 5-10 minutes; it was not accompanied by tinnitus, change of her hearing, or other neurological symptoms. There was no nausea or vomiting. After the vertigo subsided the patient continued to feel light headed and off balance for a few hours. Subsequently she had many similar episodes and there were no clear precipitating factors.

On examination in October 1997 the patient had a full range of eye movements. Her horizontal smooth pursuit movements were saccadic to the left. Saccades were normal both in the horizontal and in the vertical plane. There was no spontaneous or gaze evoked nystagmus. The vestibulo-ocular reflex was absent on turning the head to the left and there was a directional preponderance (assessed from the fast phase of nystagmus) of optokinetic responses to the left. There was no positional nystagmus on Hallpike testing. The remainder of the cranial nerves were normal. Tone, power, and reflexes were normal and plantar responses were flexor. There was no sensory loss. Heel-shin-knee testing was mildly ataxic on the right with no ataxia of the upper limbs. Her gait was unsteady and she tended to fall on Romberg's testing.

Neuro-otological examination disclosed a The stapedius reflexes were absent on the left to stimulation ipsilaterally and contralaterally - that is, consistent with facial nerve damage. Otoacoustic emissions were normal bilaterally but suppression was abnormal with a noise in the left ear and probe in the right - that is, consistent with a left 8th nerve deficit. Auditory evoked brain stem responses were absent on the left. There was a left sided canal paresis on caloric testing and electronystagmography confirmed the clinical derangement of vestibular and ocular motor function. There were occasional beats of spontaneous horizontal nystagmus to the right and left in the dark and absent vestibulo-ocular reflex suppression on turning the head to the left. The gadolinium enhanced MRI of the brain showed a heterogeneously enhancing intrapontine lesion involving the left cerebellar peduncle and extending inferiorly into the medulla, suggestive of a pontine glioma. In 
February 1998 an open brain biopsy of the lesion was attempted. The biopsy $(8 \times 5 \times 2 \mathrm{~mm})$ disclosed only normal cerebellar tissue. There was no evidence of neoplasia.

A month after the biopsy the patient developed episodic blurring of vision and a feeling that her eyes were moving. These episodes lasted a few seconds and occurred about once/minute. She was unable to read due to the intermittent oscillopsia. There were no other new neurological symptoms. When reviewed in April 1999, a video-oculogram was obtained (fig $1 \mathrm{~A}$ and B). On primary gaze there was a right over left skew deviation with spontaneous low amplitude horizontal nystagmus to the right with an exponentially increasing slow phase velocity (fig $1 \mathrm{~A}$ ). At intervals of about 40-50 seconds the upper pole of the eyes torted towards the patient's right shoulder into a tonic left over right skew over a few seconds before a coarse torsional nystagmus with fast phases carrying the upper pole of the eye to the left shoulder was initiated. The paroxysmal left beating nystagmus lasted about 12 seconds and showed a characteristic crescendo-decrescendo sequence. The eyes then slowly returned to the previous right over left skew and the horizontal right beating nystagmus immediately recurred without a clear null phase (fig $1 \mathrm{~B}$ ). The direction of gaze did not have an influence on the nystagmus amplitude or duration. This whole cycle of alternating skew deviation and direction changing nystagmus repeated itself about once every minute. During the paroxysmal
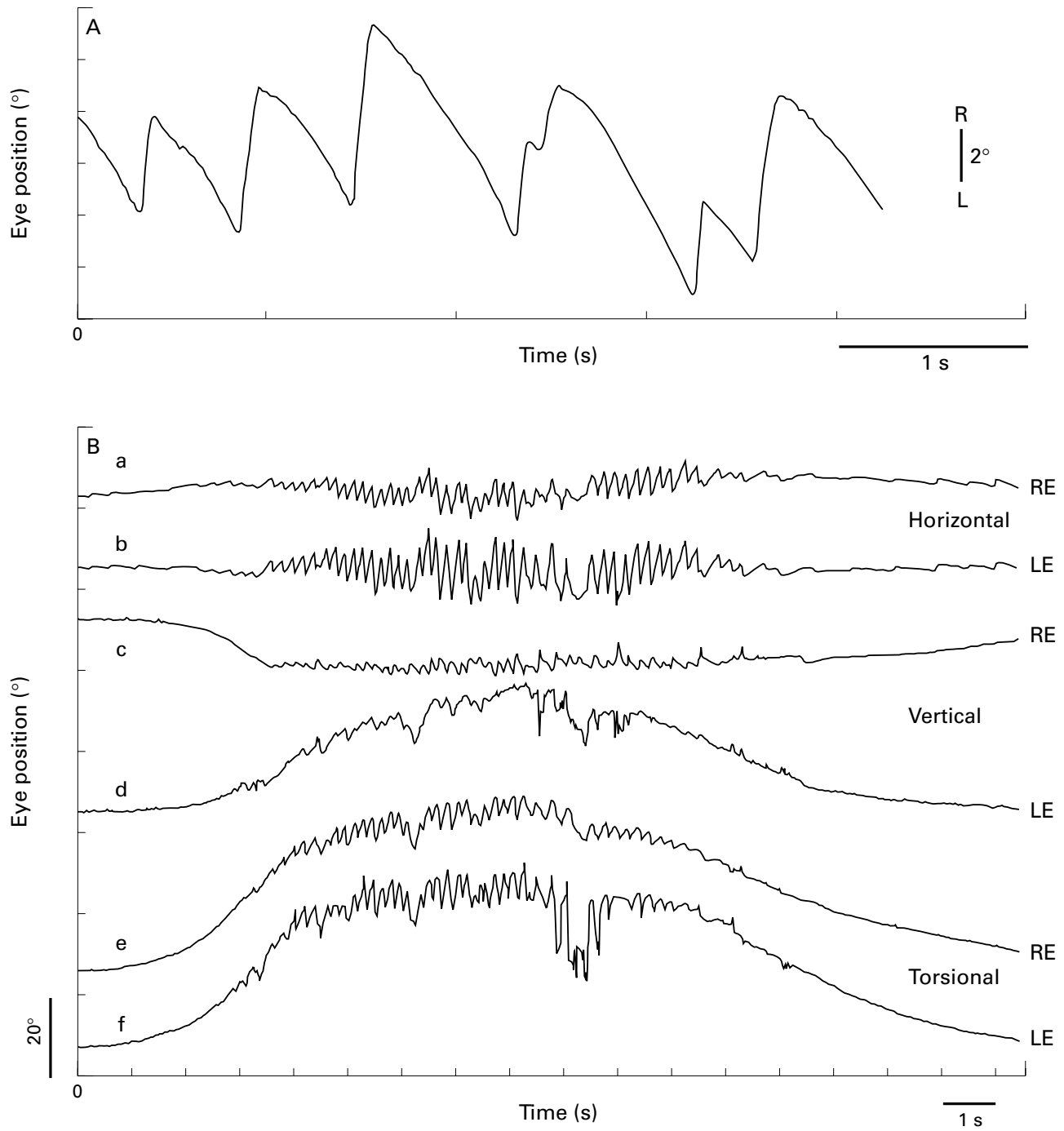

Figure 1 (A) Video-oculography (VOG: SMI, Berlin) recording of the horizontal movements of the patient's right eye during primary gaze in the dark. The trace shows right beating nystagmus with increasing slow phase velocity. $(B)$ Binocular 3 D VOG recordings of the patient's paroxysmal nystagmus. Upward tracing indicates horizontal eye movement to the right, vertical eye movement upward, and torsional roll of the upper pole of the eye to the right shoulder ("torsion to the right shoulder"). RE=right eye, $L E=$ left eye. At the beginning of the paroxysm, the right eye moves downwards and the left eye upwards into a left over right skew deviation (traces $c$ and d). The paroxysmal nystagmus is predominantly horizontal (leftbeating, traces $a$ and $b$ ) and torsional (beating towards the left shoulder, traces $e$ and $f$ ), with a slight vertical component which is upbeating in the right eye (trace $c$ ) and down beating in the left eye (trace d). Towards the end of the paroxysm the eyes drift slowly back into the previous right over left skew deviation and the horizontal nystagmus reverses direction to the baseline horizontal right beating nystagmus. The VOG system measures horizontal and vertical eye position from the displacement of the centre of the pupil in a video image of the spatient's eye. The torsional eye position is given by the rotation of the iris pattern about the pupil centre. The data sampling frequency is limited to $50 \mathrm{~Hz}$ by the video field rate. The VOG system has a linearity error of less than $1^{\circ}$ over horizontal and vertical ranges of $\pm 30^{\circ}$, and less than $0.3^{\circ}$ for torsional rotations over $\pm 30^{\circ}$. Our data has an accuracy of about $0.3^{\circ}$, with occasional artifacts when the pupil detection is affected by eyelid closure. 

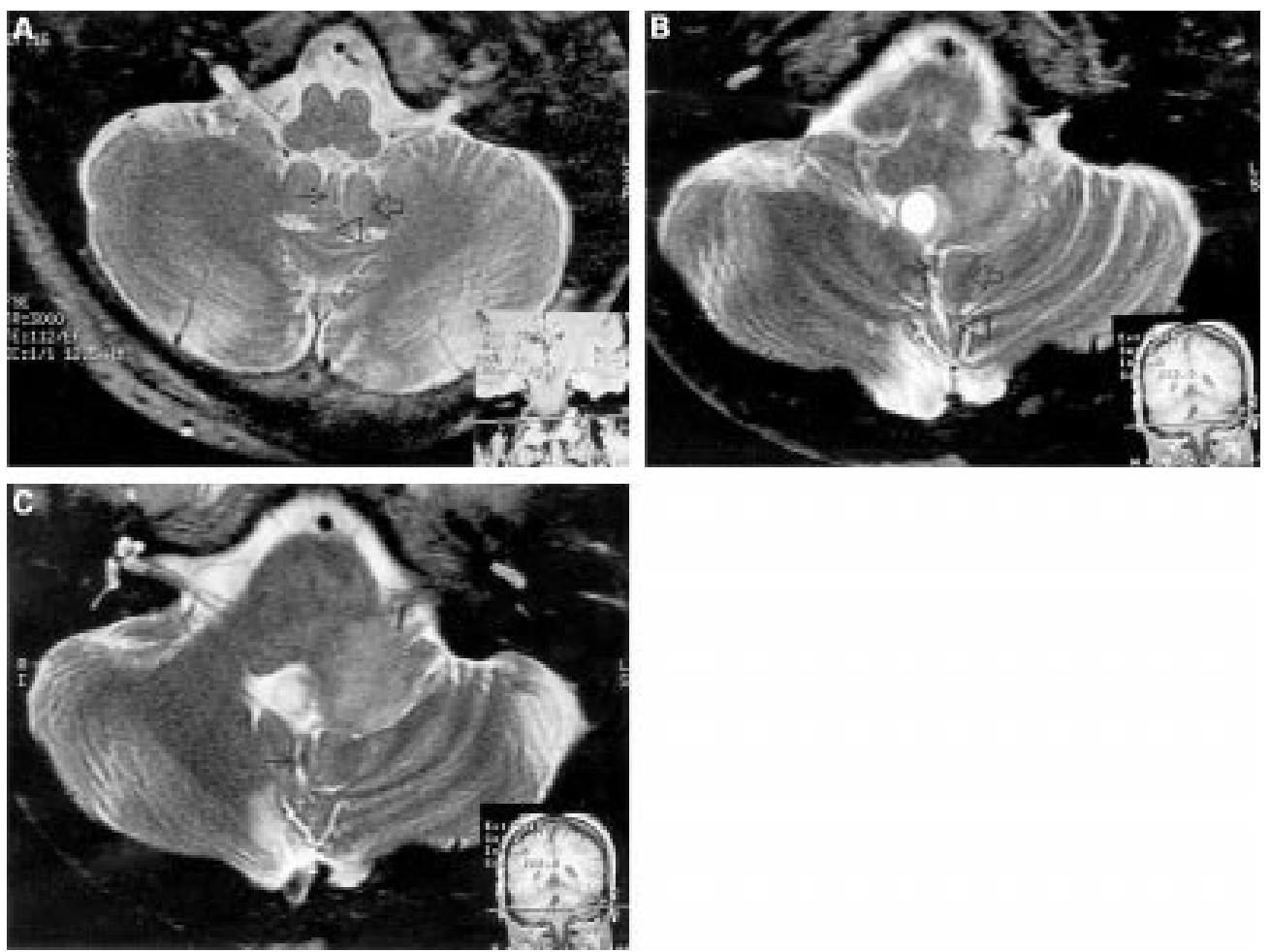

Figure 2 (A) Normal subject. Axial MRI showing tonsils (open arrow) and uvula (arrow). The pyramid lies posterior to the uvula (arrow head). (B) Axial MRI of patient at about the same level as in A. Distortion caused by the tumour and slight tilt anteriorly means that the lowest portion of the pons appears anteriorly to the medullary pyramids but the inferior vermis structures are at the same level as the normal subject. The uvula is almost completely destroyed (arrow), only the most anterior folia being spared. The extreme anterior/inferior part of the pyramid is also damaged (arrow head). Tonsils are indicated (open arrow). (C) Axial MRI of patient, $3 \mathrm{~mm}$ higher than in B demonstrating linear tract from biopsy needle throughout the uvula (arrow).

attacks of left beating nystagmus the patient was unable to read or fixate a target.

An MR scan of the posterior fossa (fig $2 \mathrm{~B}$ and $\mathrm{C}$ ) showed an intrapontine lesion, the appearance of which was the same as in the MR scan done 2 years previously. The biopsy site in the cerebellum was visualised and involved the inferior part of the uvula and probably pyramis but spared the nodulus.

\section{Discussion}

This patient developed a complex eye movement disorder with paroxysms of alternating skew deviation and direction changing nystagmus, occurring once a minute, after an unsuccessful biopsy of a brain stem lesion which resulted in the removal of a small piece of normal cerebellar tissue from the inferior uvula and adjacent pyramis. Her eye movements showed a combination of unusual features that have not previously been described:

- A resting nystagmus between attacks with exponential increase in the slow phase velocity typical of the waveform observed in congenital nystagmus.

- Episodic reversal of skew deviation at regular intervals.

- Associated attacks of paroxysmal direction changing nystagmus.

- The abnormalities occurred after a well defined surgically induced lesion of the uvula.

Paroxysmal skew deviation with nystagmus is rare. The abnormalities of interest in our patient compared with the few in the literature are the constant frequency of alternation, the asymmetry of the abnormalities, and the absence of a null point and interval between the right and left beating nystagmus. There are four pertinent case reports relating to these features. Hedges and $\mathrm{Hoyt}^{2}$ described a patient with a left upper brain stem abscess in whom right over left tonic skew deviation was associated with clockwise rotatory nystagmoid jerks lasting for 3-5 minutes, followed by a quiescent period of 30 seconds to 2 minutes. Rabinowitz et $a l^{\beta}$ described paroxysmal skew deviation with concurrent attacks of elliptical pendular oscillations of variable duration in a patient with multiple sclerosis. By contrast with these findings periodic alternating nystagmus (PAN) is usually of constant periodicity. Lewis and Kline $^{1}$ described a case of PAN where the periodically changing nystagmus was associated with periodic alternating skew deviation. However, our patient's nystagmus differed from classic PAN in that both phase length and amplitude of the nystagmus were asymmetric and there was no clear null phase intervening before the nystagmus reversed. Finally, Lawden $e t a \bar{l}$ described a patient with an arteriovenous malformation causing damage to the left vestibular nuclei, in whom attacks of paroxysmal torsional horizontal and vertical nystagmus to the left occurred regularly at 2 minute intervals lasting 15 seconds and causing oscillopsia. Between attacks there was an asymptomatic resting nystagmus in the opposite direction. This case differed from ours, however, in that there was no associated alternating skew deviation. Thus, none of these 
reports described the same phenomena seen in our patient.

What are the pathophysiological mechanisms of the eye movement abnormalities seen in our patient? The right over left skew deviation and asymptomatic right beating nystagmus are consistent with left sided vestibular hypofunction due to a destructive process in the area of the left vestibular nuclei. Lesions in the area of the medial and lateral vestibular nuclei and proximal vestibular fasciculus can cause the clinical picture similar to that of semicircular canal paresis. ${ }^{7}$ However, nystagmus in such patients is saw toothed in pattern, with constant velocity slow phases. On the other hand, nystagmus due to cerebellar lesions typically shows a decrease in slow phase velocity. However, cases have been described where acquired cerebellar dysfunction generated nystagmus with exponentially increasing slow phase velocity indistinguishable from congenital nystagmus in both the horizontal ${ }^{8}$ and verti$\mathrm{cal}^{9}$ plane. The findings in our patient, who had a surgically induced cerebellar lesion, confirm these findings that accelerating slow phase velocity can occur in acquired cerebellar damage.

The tonic reversal of the skew deviation, accompanied by reversal of direction and exacerbation of nystagmus, may be explained as a transient hyperactivity of the left vestibular nuclei. Tonic skew deviations can result from both stimulation and lesioning of peripheral and central vestibular pathways and may reflect a functional vestibular asymmetry at the level of the utricle, the vestibular nuclei in the pontomedullary region, and midbrain structures such as the interstitial nucleus of Cajal. ${ }^{10-13}$ Episodically alternating skew deviations have been described as a result of lesions along those same pathways. ${ }^{6}{ }^{14-16}$ Similarly, paroxysmal nystagmus that reverses direction is thought to be caused by an imbalance of excitatory and inhibitory activity within the vestibular system. It is possible that paroxysmal neuronal discharge occurs in damaged but otherwise hypoactive vestibular neurons. Such a mechanism was posited by Lawden et $a \bar{l}$ in their patient with an arteriovenous malformation; this patient responded to carbamazepine, suggesting a stabilisation of sodium channels.

An alternative hypothesis is that damage to the cerebellum resulted in loss of inhibitory influences upon the vestibular nuclei. The vestibular nuclei are extensively linked by both excitatory and inhibitory commissural connections. ${ }^{17}$ They are normally under ipsilateral inhibitory control of the cerebellum. ${ }^{18}{ }^{19}$ It has been shown that removal of cerebellar inhibition of vestibular nuclei may result in alternating activity in the vestibular system: lesions of the cerebellar nodulus and uvula increase the half life of the vestibular storage mechanism in the monkey and can generate periodic alternating nystagmus although only on removal of fixation. ${ }^{20}$ In our case the nodulus was spared and the inferior part of the uvula and adjacent pyramis destroyed suggesting that the uvula is the critical component in this type of nystagmus. The proposed mechanism for generation of rhythmical activity in neurons connected by inhibitory synapses is the phenomenon of postinhibitory rebound excitability, which results in periodically alternating excitation of the two cells. Removal of higher level inhibition of such a neuronal system may set off reciprocal excitation resulting in alternating activity. ${ }^{21}$ The paroxysms of alternating skew deviation nystagmus in our patient occurred at regular intervals in a highly stereotypical pattern and did not seem to be modified by external stimuli. Thus, self perpetuating reciprocal activation in the vestibular system as a result of loss of cerebellar inhibition seems to be the most likely pathogenic mechanism.

1 Lewis JM, Kline LB. Periodic alternating nystagmus associated with periodic alternating skew deviation. 7 Clin Neuroophthalmol 1983;3:115-17.

2 Hedges TR, Hoyt WF. Ocular tilt reaction due to an upper brainstem lesion: paroxysmal skew deviation, torsion, and
oscillation of the eyes with head tilt. Ann Neurol 1982;11:537-40.

3 Rabinovitch HE, Sharpe JA, Sylvester TO. The ocular tilt reaction. A paroxysmal dyskinesia associated with elliptical nystagmus. Arch Ophthalmol 1977;95:1395-8.

4 Rosengart A, Hedges TR III, Teal PA, et al. Intermittent downbeat nystagmus due to vertebral artery compression. Neurology. 1993;43:216-18.

5 Lawden MC, Bronstein AM, Kennard C. Repetitive paroxysmal nystagmus and vertigo. Neurology 1995;45:276-80.

6 Mitchell JM, Smith JL, Quencer RM. Periodic alternating skew deviation. $\mathcal{F}$ Clin Neuroophthalmol 1981;1:5-8.

7 Francis DA, Bronstein AM, Rudge P, et al. The site of brainstem lesions causing semicircular canal paresis: an

8 Barton JJS, Sharpe JA. Oscillopsia and horizontal nystagmus with accelerating slow phases following lumbar puncmus with accelerating slow phases following lumbar punc33:418-21.

9 Zee DS, Leigh JL, Mathieu-Millaire F. Cerebellar control of ocular gaze stability. Ann Neurol 1980;7:37-40.

10 Brandt T, Dieterich M. Pathological eye-head coordination in roll: tonic ocular tilt reaction in mesencephalic and medullary lesions. Brain. 1987;110:649-66.

11 Halmagyi GM, Curthoys IS, Brandt T, et al. Ocular tilt reaction: clinical sign of vestibular lesion. Acta Otolaryngol Suppl 1991;481:47-50.

12 Brandt T, Dieterich $M$. Skew deviation with ocular torsion: a vestibular brainstem sign of topographic diagnostic value. Ann Neurol 1993;33:528-34.

13 Lueck CJ, Hamlyn P, Crawford TJ, et al. A case of ocular tilt reaction and torsional nystagmus due to direct stimulation of the mid-brain in man. Brain 1991;114:2069-79.

14 Corbett JJ, Schatz NJ, Shults WT, et al. Slowly alternating skew deviation: description of pretectal syndrome in three patients. Ann Neurol 1981;10:540-6.

15 Keane JR. Alternating skew deviation: 47 patients. Neurology 1985;35:725-8.

16 Bentley CR, Bronstein AM, Faldon M, et al. Fast eye movement initiation of ocular torsion in meso-diencephalic lesions. Ann Neurol 1997;43:729-37.

17 Brodal A. Organization of the commissural connections: anatomy. Prog Brain Res 1972;37:167-76.

18 Ito $M$. Cerebellar control of the vestibular neurons: physiology and pharmacology. Prog Brain Res 1972;37:377-90.

19 Barr CC, Schultheis LW, Robinson DA. Voluntary, nonvisual control of the human vestibulo-ocular reflex. Acta Otolaryngol 1976;81:365-75.

20 Waespe W, Cohen B, Raphan T. Dynamic modification of the vestibulo-ocular reflex by the modulus and uvula. Science 1984;228:199-202.

21 Perkel DH, Moloney B. Motor pattern production in reciprocally inhibitory neurons exhibiting post-inhibitory rebound. Science 1974;185:181-3. 